\title{
Linear IgA bullous disease presenting as a Hailey-Hailey disease associated with Hashimoto thyroiditis: a case report
}

\section{Katarzyna Tomaszewska1,2, Magdalena Oszukowska1, Anna Zalewska-Janowska², Andrzej Kaszuba ${ }^{1}$}

${ }^{1}$ Department of Dermatology, Pediatric Dermatology and Oncology, Medical University of Lodz, Lodz, Poland, ${ }^{2}$ Department of Psychodermatology, Chair of Clinical Immunology and Rheumatology, Medical University of Lodz, Lodz, Poland

Corresponding author: Dr. Katarzyna Tomaszewska, E-mail: tomaszewska.katarzyna@o2.pl

\begin{abstract}
Linear IgA bullous disease (LABD) is a rare autoimmune subepidermal bullous disease characterized by linear deposits of IgA anti-basement membrane zone antibodies. The skin lesions are variable: papular, vesicular, bullous, erythematous, edematous, and erythema multiforme-like, mimicking bullous pemphigoid, dermatitis herpetiformis, toxic epidermal necrolysis, mucous membrane pemphigoid or in described case - Hailey-Hailey disease. We present a case of a 17-year-old female patient diagnosed with LABD associated with Hashimoto thyroiditis
\end{abstract}

Key words: Autoimmune disease; Bullous disease; Clinical dermatology

\section{INTRODUCTION}

Linear IgA bullous disease (LABD) is a rare autoimmune subepidermal bullous disease characterized by linear deposits of IgA anti-basement membrane zone antibodies. Most cases of LABD are idiopathic and the etiology of the disease remains unclear, but some cases are occasionally induced by drugs, internal malignancies, and infections [1].

\section{CASE REPORT}

A previously healthy 17-year-old female patient presented with a 5-months history of vesicular eruptions. Physical examination revealed multiple vesicles and bullae on an erythematous base. First eruptions appeared in axillae and groins. The initial diagnosis was Hailey-Hailey disease. Topical treatment was established with gradual progression of the disease. The lesions spread on the lower trunk. back thigh surfaces, neck, facial skin around the mouth. The typical bullous eruption with a 'string of pearls' sign appeared on left forearm. Oral mucosa, eyes, and nails were spared. General condition was good.

The patient's blood test revealed elevated antibodies against thyroid peroxidase (TPO) and antibodies against thyroglobulin (TG). The thyroid stimulating hormone (TSH) was $4,88 \mathrm{mU} / \mathrm{L}(0,27-4,2)$, with a free triiodothyronine (fT3) of 2,6 pmol/L (3,1-6.8) and a free thyroxine (fT4) of $9.6 \mathrm{pmol} / \mathrm{L}(12.0-22.0)$. Other laboratory studies including hematological examination, blood biochemistry, and urine analysis, antinuclear antibodies were normal. The family history of autoimmune diseases was negative.

Direct immunofluorescence (DIF) of biopsy specimens of normal skin demonstrated linear IgA-deposit pattern at the dermo-epidermal junction (DEJ) in the absence of other immunoglobulins and complement. Indirect immunofluorescence of serum was negative.

With the above findings, a diagnosis of LABD and Hashimoto thyroiditis was made. The patient was

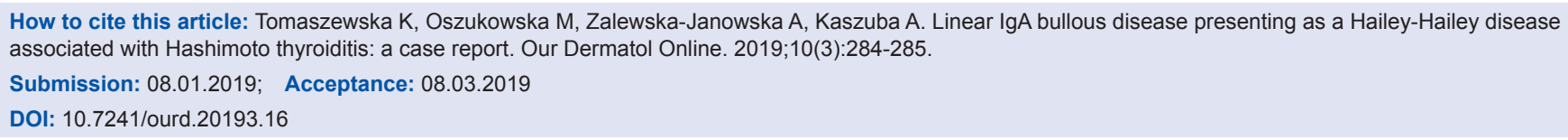




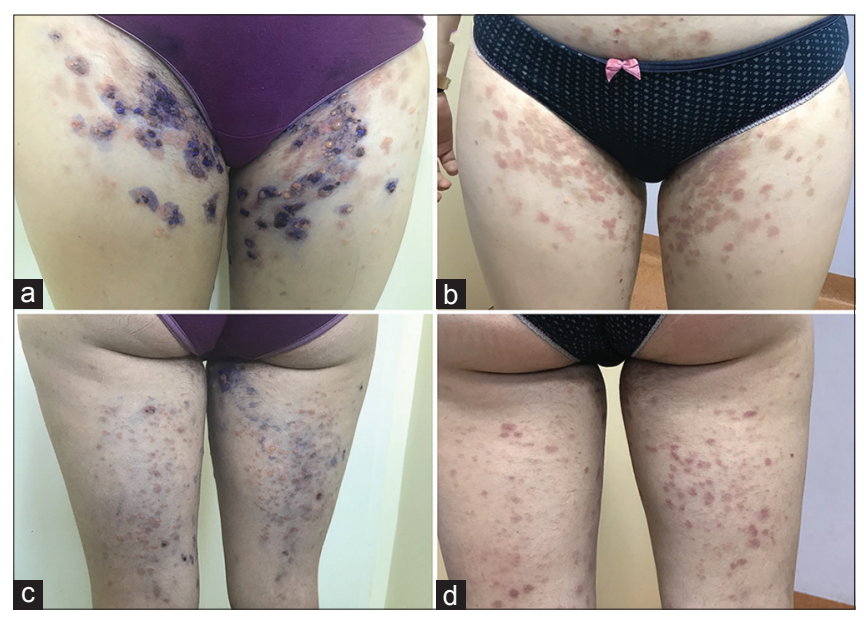

Figure1: Multiple vesicles and bullae on an erythematous base in groins ( $a$ - before treatment; $b$ - 3 weeks after starting an oral course of dapsone $50 \mathrm{mg}$ per day) and back thigh surfaces (c - before treatment; $\mathrm{d}-3$ weeks after starting an oral course of dapsone $50 \mathrm{mg}$ per day).

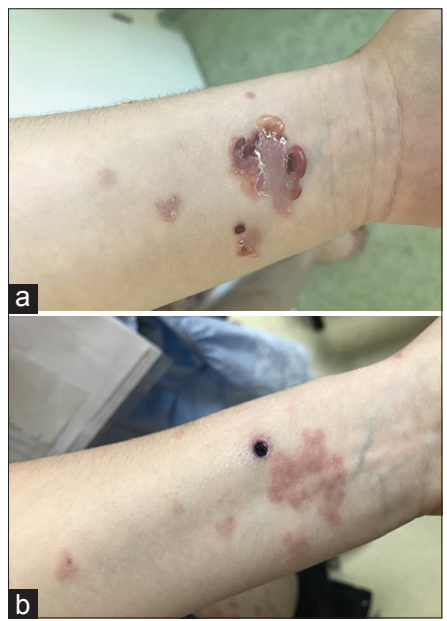

Figure 2: The typical bullous eruption with a 'string of pearls' sign on left forearm ( $\mathrm{a}$ - before treatment; $\mathrm{b}$ - 3 weeks after starting an oral course of dapsone $50 \mathrm{mg}$ per day).

started on a course of oral dapsone $50 \mathrm{mg}$ daily to which her skin lesions responded (Figs. 1 and 2). The treatment was well tolerated and produced no side effects. The patient is under the care of the Dermatology and Endocrinology Departments.

\section{DISCUSSION}

LABD is defined according to the three following clinical and histological criteria: vesicular or bullous eruption involving skin and mucous membranes (MMs), subepidermal blisters infiltrated predominantly by neutrophils in lesion biopsies and a linear IgA-deposit pattern at DEJ in the absence of other immunoglobulins on DIF [2].

The typical clinical picture is a bullous eruption with a 'string of pearls' sign, especially in childhood. The skin lesions in adult LABD are variable: papular, vesicular, bullous, erythematous, edematous, and erythema multiforme-like, mimicking bullous pemphigoid (BP), dermatitis herpetiformis (DH), toxic epidermal necrolysis or mucous membrane pemphigoid (MMP) [3].

In the described case, the symmetric distribution was confined to the areas typical of Hailey-Hailey disease (areas exposed to friction such as the sides of the neck, axillae, and groins) [4]. The adolescence-onset of disease was also similar to Hailey-Hailey disease.

Furthermore, the patient was diagnosed with Hashimoto thyroiditis which is also an autoimmune disease. Autoimmune diseases are common conditions which appear to develop in genetically susceptible individuals, with an expression of the disease being modified by permissive and protective environments [5].

\section{Consent}

The examination of the patient was conducted according to the Declaration of Helsinki principles.

\section{REFERENCES}

1. Ishii N. Prognostic factors of patients with linear IgA bullous dermatosis. Br J Dermatol. 2017;177:16-7.

2. Abreu Velez AM, Calle J, Howard MS. Autoimmune epidermal blistering diseases. OurDermatol Online. 2013;4(Suppl.3):631-46.

3. Gottlieb J, Ingen-Housz-Oro S, Alexandre M, Grootenboer-Mignot S, Aucouturier F, Sbidian E, et al. Idiopathic linear IgA bullous dermatosis: prognostic factors based on a case series of 72 adults. Br J Dermatol. 2017;177:212-2.

4. Al Hamzawi NK. Hailey-Hailey disease presenting at unusualsite. Our Dermatol Online. 2019;10:108-9.

5. Forabosco P, Bouzigon E, Ng MY, Hermanowski J, Fisher SA, Criswell LA, et al. Meta-analysis of genome-wide linkage studies across autoimmune diseases. Eur J Hum Genet. 2008;17:236-43.

Copyright by Katarzyna Tomaszewska, et al. This is an open-access article distributed under the terms of the Creative Commons Attribution License, which permits unrestricted use, distribution, and reproduction in any medium, provided the original author and source are credited.

Source of Support: Nil, Conflict of Interest: None declared. 